\title{
A corrector for spacecraft calculated electron moments
}

\author{
J. Geach ${ }^{1,2}$, S. J. Schwartz ${ }^{1,3}$, V. Génot ${ }^{4}$, O. Moullard ${ }^{1}$, A. Lahiff ${ }^{5}$, and A. N. Fazakerley ${ }^{5}$ \\ ${ }^{1}$ Space Plasma Group, Astronomy Unit, Queen Mary, University of London, Mile End Road, London E1 4NS, UK \\ ${ }^{2}$ now at: Department of Physics, University of Durham, Durham DH1 3HP, UK \\ ${ }^{3}$ now at: Space and Atmospheric Physics, Blackett Laboratory, Imperial College London, London SW7 2AZ, UK \\ ${ }^{4}$ Centre d'Etude Spatiale des Rayonnements, 9 Avenue du Colonel Roche, Boite Postale 4346 31028, Toulouse, France \\ ${ }^{5}$ Mullard Space Science Laboratory, University College London, Holmbury St. Mary, Dorking, Surrey RH5 6NT, UK
}

Received: 26 August 2004 - Revised: 31 January 2005 - Accepted: 2 February 2005 - Published: 30 March 2005

\begin{abstract}
We present the application of a numerical method to correct electron moments calculated on-board spacecraft from the effects of potential broadening and energy range truncation. Assuming a shape for the natural distribution of the ambient plasma and employing the scalar approximation, the on-board moments can be represented as non-linear integral functions of the underlying distribution. We have implemented an algorithm which inverts this system successfully over a wide range of parameters for an assumed underlying drifting Maxwellian distribution. The outputs of the solver are the corrected electron plasma temperature $T_{e}$, density $N_{e}$ and velocity vector $\boldsymbol{V}_{e}$. We also make an estimation of the temperature anisotropy $A$ of the distribution. We present corrected moment data from Cluster's PEACE experiment for a range of plasma environments and make comparisons with electron and ion data from other Cluster instruments, as well as the equivalent ground-based calculations using full 3-D distribution PEACE telemetry.
\end{abstract}

Keywords. Space plasma physics (Experimental and mathematical techniques; Spacecraft sheaths, wakes, charging; Instrumentation and techniques)

\section{Introduction}

The space physicist - interested in the moments of the electron distribution around the Earth - must strike a balance between the advantages and disadvantages of on-board calculations of the moments. Under most circumstances there is insufficient telemetry to transmit samples from the distribution function itself at a high time resolution, but there is ample bandwidth to transmit the moments, which encode basic information about the distribution in just a few numbers.

Moments calculated on-board spacecraft typically over- or under-estimate the values of the "true" moments, because they convolve effects caused by the presence of a potential

Correspondence to: S. J. Schwartz

(s.schwartz@imperial.ac.uk) (from the spacecraft itself) and lower and upper energy truncation imposed by the detector. Furthermore, the plasma environment determines the formation of photo- and secondary electrons, which can return to the spacecraft and enter the detector, therefore contaminating the measured moments. Conversely, full-distribution telemetry can be treated on the ground, using more sophisticated computation than is available on the spacecraft.

Generation of spacecraft potential is determined by the balance of the currents flowing away from the craft carried by liberated electrons, and the incident electrons and ions from the ambient plasma. The value of the potential is therefore determined by the plasma environment, specifically the density and temperature (Pedersen, 1995; Escoubet et al., 1997). Attempts have been made to dynamically control the potential, such as ASPOC (Active Spacecraft POtential Control) on three of the Cluster spacecraft (Riedler et al., 1997), which aims to stabilise the potential by emitting a positive (indium) ion beam. Devices such as ASPOC can limit the build up of potential (which can reach values of $70 \mathrm{~V}$ ) to just a few Volts (Schmidt et al., 1995), and in general aim to constrain the potential to less than $10 \mathrm{~V}$ (Torkar et al., 2001). While the charging of the spacecraft can be limited, no real detector is free from the constraints of a finite energy range and calibration defects, the latter of which are very difficult to correct after convolution by the on-board calculation. All on-board calculated moments must therefore be treated with caution.

Song et al. (1997) present the concept of a perfect plasma detector, which is free from calibration defects, for which the uncertainties in the on-board moments are solely caused by the spacecraft potential, a truncated energy range and the presence of secondary and photo-electrons. Those authors indicate that in the case of electrons, the lower energy cutoff should be calculated as the detector's nominal lower energy limit minus the spacecraft potential. However, Song et al. (1997) do not pursue further the impact of a non-zero spacecraft potential on the distribution function arriving at the spacecaft. They restrict their results to the case of a drifting distribution truncated by a detector but with a null 
spacecraft potential. Salem et al. (2001) showed how both the integration limits and, via Liouville's Theorem, modifications to the distribution function in the presence of a non-zero potential need to be taken into consideration. They applied their formalism to the case of a non-drifting Maxwellian under conditions typical of the solar wind.

Génot and Schwartz (2004, hereafter GS) extend this idea of a perfect detector to the drifting case under a variety of solar wind and magnetospheric conditions. They present a method to disentangle the effects of potential and energy range truncation using a non-linear numerical routine (though it is important to note that this method does not correct for the contamination of secondary electrons - for a discussion on this topic see Szita et al., 2001). GS demonstrate that the measured moments can be expressed as functions of the true moments and the spacecraft potential, where the true moments are those which would be measured by a perfect detector. We implement the technique proposed by GS such that, given a set of measured moments and knowledge of the spacecraft potential and detector limits, the true moments can be recovered; we call these the corrected moments.

The magnitude of the difference between the on-board and corrected moments is a function of both environment and potential. GS show that the solar wind is a region where the moments are seriously affected (see also Salem et al., 2001). For a potential ranging from zero to 10 Volts, the density can be under-estimated by $60 \%$ for low potentials to overestimation of $75 \%$ for high potentials. In general the other moments are over-estimated. Those authors make the interesting point that there exists a critical potential for which the on-board density moment equals the corrected one (see GS and Salem et al., 2001), though no such regimes exist for the other moments for typical plasma environments. In other regions such as the magnetosheath and magnetosphere, the moments are less severely compromised as in the solar wind, but the effects there are by no means negligible, with up to a $40 \%$ under-estimation of the density in the magnetosheath and $10 \%$ in the magnetosphere during nominal operating conditions. Fundamentally the presence of a potential affects the width of the distribution function, such that for a naturally broad (i.e. hot) distribution, the extra broadening caused by spacecraft effects is slight; the opposite is true for cool distributions.

In this paper we show that it is possible to correct on-board calculated moments from a wide range of environments with a high success rate and in excellent agreement to ground based calculations and data from other instruments. Although the algorithm we present is general to any on-board moments, we have tested extensively on data taken by Cluster's PEACE (Plasma Electron and Current Experiment) instrument (Johnstone et al., 1997). PEACE calculates moments on-board every spacecraft spin $(\sim 4 \mathrm{~s})$, and transmits the full 3-D distrubution at high resolution as frequently as the telemetry allocation will allow. During burst mode operation this can be also at spin resolution, providing an excellent opportunity to compare on-board corrected moments using our routine to ground calculated ones. We make comparisons with other measurements from both plasma and wave instruments on Cluster to further verify our results, and present the results of a mapping survey of Cluster data over a wide range of environments to show the distribution (in density-temperature parameter space) of (a) the global deviation of the on-board moments, (b) electron bulk velocity, (c) spacecraft potential and (d) a simulation of the expected deviation from the true moments.

In Sect. 2 we discuss the definitions of the moments; in Sect. 3 we present the numerical method behind the algorithm; in Sect. 4 we present an overview of results from a survey of PEACE data; in Sect. 5 we make comparisons of corrected moments with those derived from the full 3-D ground integration and with other instruments; in Sect. 6 we discuss the limitations of, and possible improvements to our method; in Sect. 7 we briefly summarise the correcting procedure and in Sect. 8 we make our conclusions.

\section{Moments}

\subsection{Definitions}

The nth moment of a distribution $f(\mathbf{v})$ is defined as:

$\mathbf{M}_{n}=\int f(\mathbf{v}) \mathbf{v}^{n} \mathrm{~d}^{3} v$

Certain combinations of the moments from an electron velocity distribution have a familiar physical interpretation:

$N=M_{0}$

$N \boldsymbol{V}=\boldsymbol{M}_{1}$

$\mathbf{P}=m_{e} \mathbf{M}_{\mathbf{2}}$

$\mathbf{p}=\mathbf{P}-m_{e} \mathbf{M}_{\mathbf{1}} \boldsymbol{V}$

where $N$ is the density, $\boldsymbol{V}$ the bulk velocity vector, $\mathbf{P}$ the stress tensor and $\mathbf{p}$ the pressure tensor. We assume that in free space (i.e. far away from the spacecraft where there are no effects from the potential), the distribution has a Maxwellian shape at temperature $T$, and drifts with some velocity $\boldsymbol{V}$ :

$f(\mathbf{v})=N\left(\frac{m_{e}}{2 \pi k T}\right)^{3 / 2} \exp \left(-\frac{m_{e}}{2 k T}|\mathbf{v}-\boldsymbol{V}|^{2}\right)$

Integration over all solid angles and energies yields the number density $N$. It is easy to see therefore that in general for a finite integration range $v_{l}<|\mathbf{v}|<v_{u}$, as used in real detectors, $N$ will be underestimated. Note that the amount the on-board moments are under- or over-estimated is also a function of spacecraft potential (see GS), such that for large potentials the density is over-estimated.

Near the spacecraft the energy conservation of an electron can be expressed:

$v_{m}^{2}=v^{2}-\mathcal{E}$ 


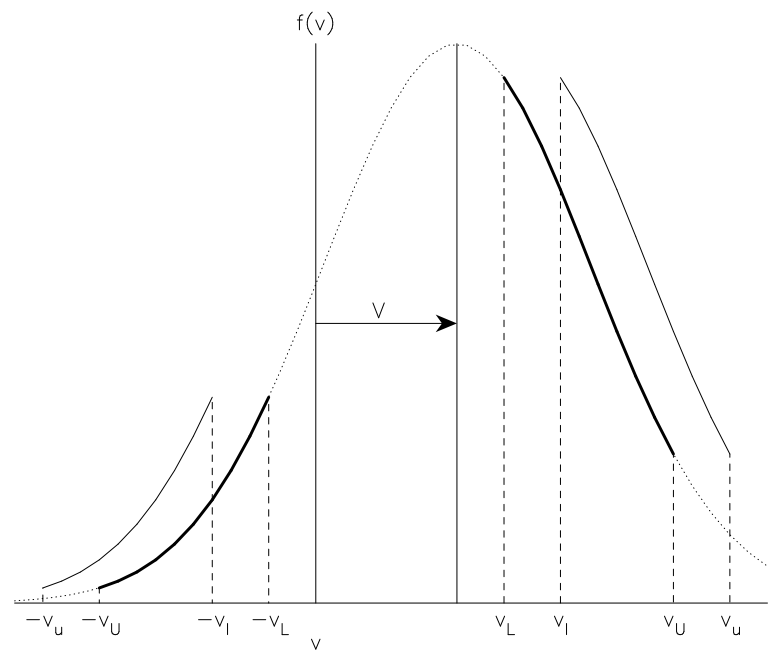

Fig. 1. Schematic showing the effect of a positive spacecraft potential on an idealised drifting Maxwellian electron distribution (dotted curve). On-board calculated moments are integrals under the shifted segments (solid grey) which can be writtten, with the help of Liouville's Theorem, in terms of the idealised distribution. Inverting this then yields the Maxwellian parameters.

where $v$ is the electron velocity in free space (hereafter a subscript $m$ denotes a parameter as measured by the spacecraft and $c$ denotes output from the solver; $s c$ is a value inherent to the spacecraft, such as potential) and $\mathcal{E}$ corresponds to the free space energy of an electron which arrives at the detector with zero energy:

$\mathcal{E}=-\frac{2 e \Phi_{s c}}{m_{e}}$.

$\mathcal{E}$ is negative for most of the plasma environments in space, since typically the spacecraft potential $\Phi_{s c}>0$ due to the escape of photo-electrons. Liouville's theorem tells us that the distribution function is constant along a phase space trajectory

$f\left(v_{m}, \theta_{m}, \phi_{m}\right)=f(v, \theta, \phi)$.

GS make the scalar approximation: namely that only the magnitude of the velocity is affected by the potential (i.e. $\theta_{m}=\theta$ and $\left.\phi_{m}=\phi\right)$. Under this assumption the angular dependence in the moment integrations can be performed analytically, thus reducing the problem to one dimension. By changing the integration element $v_{m} \mathrm{~d} v_{m}=v \mathrm{~d} v$ from Eq. (7), the measured moments can be written in terms of $v$ and $\mathcal{E}$. The integration limits are related to the detector cut-offs $v_{l, u}$ by

$v_{L, U}=\sqrt{v_{l, u}^{2}+\mathcal{E}}$.

In the case of $v_{l}^{2}+\mathcal{E}<0$ where the potential reaches a value greater than the lower energy cut-off, we set $v_{L}=0$. Figure 1 is a schematic representation of the measured part of the distribution compared to the corrected part and the underlying (assumed) Maxwellian distribution.
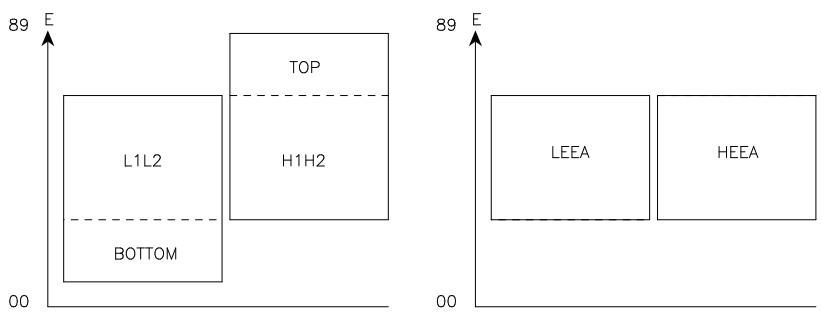

Fig. 2. Schematic representation of the PEACE sensor coverage. The two sensor heads HEEA and LEEA are split into an energy range which is divided into 88 bins. The sensor coverages can overlap by some amount (left), leaving a low energy portion (BOTTOM) and a high energy portion (TOP). The sensors can be made to overlap completely (right) leaving no BOTTOM or TOP moments.

\subsection{PEACE moment sums}

The Cluster PEACE experiment is made up of two sensor heads called LEEA and HEEA (Low and High Energy Electrostatic Analysers), mounted on opposite sides of each of the four Cluster spacecraft (Johnstone et al., 1997). Generally LEEA scans the lower energy range and HEEA the upper range, together covering electrons with $0.7 \mathrm{eV}<E<26 \mathrm{keV}$. Over the duration of a spin $(\sim 4 \mathrm{~s})$, LEEA and HEEA cover $4 \pi$ steradians of velocity space.

The moment sums can be thought of as combinations of the low energy moments (the B (BOTTOM) moments, $E \sim 10 \mathrm{eV}$ ), the high energy moments (the T (TOP) moments, $E \gtrsim 2 \mathrm{keV}$ ), and the moments from where the energy scan of LEEA and HEEA overlap (which we call L1L2 and H1H2 respectively) as sketched in Fig. 2. These separate pieces are telemetered to the ground, where we can construct moment sums covering the entire energy range by summing B, L1L2/H1H2 and T moments. The "measured" moments (subscript $m$ ) are the raw, uncorrected sum of, for example $\mathrm{B}+$ overlap+T moments, which have not been corrected.

In practice, if the B moments commence within a user controllable threshold of the spacecraft potential, they are excluded from the moment sum. This minimises the impact of contamination due to photo- and/or secondary electrons at the expense of raising $v_{L}$ and hence excluding a larger region of phase-space. Additionally, when inverting our non-linear system (see Sect. 3.3) we exclude the T moments.

\section{Method}

\subsection{Calculation frame}

Our choice of calculation frame simplifies greatly the numerical technique. We transform the spacecraft measurement frame via a rotation $\mathcal{R}$ which aligns the $z$ axis with the measured velocity $\boldsymbol{V}_{m}$ such that $\boldsymbol{V}_{m}=\left(0,0,\left|\boldsymbol{V}_{m}\right|\right)$. The scalar quantity $N_{m}$ remains the same, as does the trace of the pressure tensor. The transformed quantities can be directly inferred from the measured values, so the details of the rotation matrix do not need to be known. As, due to the scalar 
approximation, only the magnitude of the velocity is needed in Eqs. (11-13), we can use the measured direction of the velocity to recover the corrected velocity vector from the speed derived in our calculation.

\subsection{Inversion}

From Fig. 1 we see that for a given free space Maxwellian distribution described by $\left(N_{c}, V_{c}, T_{c}\right)$, the measured moments are integrals over the shifted and truncated regions. The objective is to invert the set of non-linear equations:

$g_{1}\left(N_{c}, V_{c}, T_{c}\right)-N_{m}=0$

$g_{2}\left(N_{c}, V_{c}, T_{c}\right)-N_{m} V_{m}=0$

$g_{3}\left(N_{c}, V_{c}, T_{c}\right)-3 N_{m} k T_{m}-m_{e} N_{m} V_{m}^{2}=0$

where the measured moments $\left(N_{m}, V_{m}, T_{m}\right)$ are described as functions of the corrected moments $\left(N_{c}, V_{c}, T_{c}\right)$. The Appendix recasts the functions $g_{i}$ derived in GS into the normalised form we implement numerically. We use a 100point Gaussian quadrature routine to handle integration and a Newton-Raphson algorithm (Press et al., 1992) for the nonlinear solver.

The algorithm converges to a solution by improving on a set of initial guesses. The values of initial guess $\left(\eta, V_{c}^{\prime}, V_{T c}^{\prime}\right)$ (see the Appendix) we use were derived from a series of tests in which, given a set of Maxwellian parameters, we simulated measured moments given a range of $\Phi_{s c}$ and energy cut-offs. We ran our algorithm on these inputs to recover the initial underlying Maxwellian values. The initial guesses were then chosen to be the average values for which the alorithm converged successfully for a range of parameter space which represents typical plasma environments encountered by spacecraft - our choice of parameter space is consistent with that tested by GS.

In some circumstances we found that the choice of initial guess was critical in reaching a convergence. If necessary, we perform a sequence of initial guesses until the NewtonRaphson scheme can proceed to a converged solution, the most sensitive parameter being the normalised density $\eta$. We found this simple technique very effective when dealing with large quantities of data spanning a range of environments. When applied to a time series of data we try the most recent successful guess prior to reverting to the more general approach described above.

\subsection{Adding high energy moments}

In practice, we restrict the inversion of Eqs. (11-13) to moment sums which exclude the TOP (i.e. higher energy) moments of PEACE. This improves the numerical accuracy and speed of the algorithm, since high energy moments do not require correcting as the presence of a finite spacecraft potential in the TOP energy regime has neglibible effect. These moments (described by the TOP moments in the case of PEACE) are incorporated into the overall sum in the following way.
Given the measured onboard moments for $v<v_{u}$, that is, $\left(N_{m},(N \boldsymbol{V})_{m}, \mathbf{P}_{m}\right)$, we use our correcting algorithm to yield the characterising features $\left(N_{c}, V_{c}, T_{c}\right)$ of the Maxwellian distribution $f(v)$ (Eq. 6). The direction of the velocity is identical to the measured velocity direction by virtue of the scalar approximation. We then perform three 1-D integrations on the system $g_{1,2,3}$ (see the Appendix for details) over the truncated range $0 \leq v \leq v_{u}$ with a null spacecraft potential to restore the corrected moments into the truncated-corrected form $\left(N_{t c},(N V)_{t c}, \operatorname{Tr}\left(\mathbf{P}_{t c}\right)\right)$ :

$N_{t c}=g_{1}\left[N_{c}, V_{c}, T_{c}, \Phi_{s c}=0\right]_{v_{L}=0}^{v_{U}=v_{u}}$

$(N V)_{t c}=g_{2}\left[N_{c}, V_{c}, T_{c}, \Phi_{s c}=0\right]_{v_{L}=0}^{v_{U}=v_{u}}$

$\operatorname{Tr}\left(\mathbf{P}_{t c}\right)=g_{3}\left[N_{c}, V_{c}, T_{c}, \Phi_{s c}=0\right]_{v_{L}=0}^{v_{U}=v_{u}}$

where

$\operatorname{Tr}\left(\mathbf{P}_{t c}\right)=3 p_{t c}+2 m_{e}(N V)_{t c} V_{c}-m_{e} N_{t c} V_{c}^{2}$

The direction of $(N V)_{t c}$ is restored by the measured velocity direction, and the corrected stress tensor is reconstructed into a velocity aligned frame thus:

$\mathbf{P}_{t c}=p_{t c} \mathbf{I}+m_{e} N_{t c}\left(2 V_{t c} V_{c}-V_{c}^{2}\right) \hat{\boldsymbol{V}}_{m} \hat{\boldsymbol{V}}_{m}$

From here the TOP moments, assumed to reflect the integrals of $f(v)$ from $v_{u} \rightarrow \infty$, can be added to the truncatedcorrected moments yield the final corrected moments:

$N_{f}=N_{t c}+N_{T}$

$(N \boldsymbol{V})_{f}=(N \boldsymbol{V})_{t c}+(N \boldsymbol{V})_{T}$

$\mathbf{P}_{f}=\mathbf{P}_{t c}+\mathbf{P}_{T}$

\subsection{Temperature anisotropy}

The simplification of the method proposed in GS and implemented here lies in the reduction to a 1-D integration, made possible through the use of the scalar approximation and an isotropic distribution. However we can attempt to estimate the anisotropy - i.e. the random velocities parallel and perpendicular to the magnetic field - and interpret the result as two temperature components $T_{\|}$and $T_{\perp}$. We make the assumption that the anisotropy is unchanged by the presence of a potential, and base our estimation on the anisotropy of the measured pressure tensor. Here we describe the method.

The $i j$ th element of the measured pressure tensor $\mathbf{p}_{m}$ can be expressed

$p_{m_{i j}}=P_{m_{i j}}-m_{e}(N V)_{m i} V_{m j}$

which can be decomposed in terms of the unit magnetic field vector $\mathbf{b}$ in the case of a gyrotropic distribution:

$p_{m_{i j}}=p_{m \|} b_{i} b_{j}+p_{m \perp}\left(\delta_{i j}-b_{i} b_{j}\right)$.

The trace of $\mathbf{p}_{m}$ is then

$\operatorname{Tr}\left(\mathbf{p}_{m}\right)=p_{m \|}+2 p_{m \perp}$. 
Finally the measured anisotropy $A_{m}$ is found as

$A_{m}=\frac{p_{m \perp}}{p_{m \|}}=\frac{\operatorname{Tr}\left(\mathbf{p}_{m}\right)-p_{m \|}}{2 p_{m \|}}$.

We make the assumption that this measured anisotropy is an approximation to the underlying anisotropy and so we can partition the corrected temperature $T_{f}$ according to:

$3 T_{f}=T_{f \|}+2 T_{f \perp}$

with

$\frac{T_{f \perp}}{T_{f \|}} \equiv A=A_{m}$

As a check we rotate $\mathbf{p}_{m}$ into a field-aligned frame such that:

$\left[\begin{array}{lll}p_{m 00} & p_{m 01} & p_{m 02} \\ p_{m 10} & p_{m 11} & p_{m 12} \\ p_{m 20} & p_{m 21} & p_{m 22}\end{array}\right] \rightarrow\left[\begin{array}{ccc}p_{m \|} & 0 & 0 \\ 0 & p_{m \perp_{1}} & 0 \\ 0 & 0 & p_{m \perp_{2}}\end{array}\right]$

In practice the off-diagonal terms are not identically zero and we use the ratio of these to the diagonal terms as an error check. Additionally $p_{m \perp 1} \neq p_{m \perp 2}$ provides a second check.

\subsection{Estimation parameters}

We calculate three estimation parameters which describe how much the measured on-board moments were over- or under-estimated:

$r_{N}=\frac{N_{m}-N_{f}}{N_{f}}$

$r_{V}=\frac{V_{m}-\left|\boldsymbol{V}_{f}\right|}{\left|\boldsymbol{V}_{f}\right|}$

$r_{T}=\frac{\operatorname{Tr}\left(\mathbf{p}_{m}\right) / 3 N_{m} k-T_{f}}{T_{f}}$

$I_{e}=\sqrt{r_{N}^{2}+r_{V}^{2}+r_{T}^{2}}$.

These parameters trace the effect that various environments have on the measured moments. A more general estimation parameter $I_{e}$ is yielded by the Pythagorean combination of the three parameters above (Eq. 31). Generally $I_{e}<1$ (that is, $<60 \%$ correction on all moments). As mentioned above, the amplitude of the over- or under-estimation is determined by environment, however their general behaviour is also a function of spacecraft potential (which itself is somewhat influenced by the characteristics of the ambient plasma). GS describe how the estimation parameters vary for a range of potentials in three plasma environments: the solar wind, magnetosphere and magnetosheath. Those authors conclude that the moments are affected worst in the solar wind, where $I_{e}$ can exceed $60 \%$. In the other regions the moments are affected to a lesser extent, but in general still require correcting. GS and Salem et al. (2001) also describe the existence of a critical potential, $\Phi_{\text {crit }}$, for which the ratio $r_{N}$ is zero, and

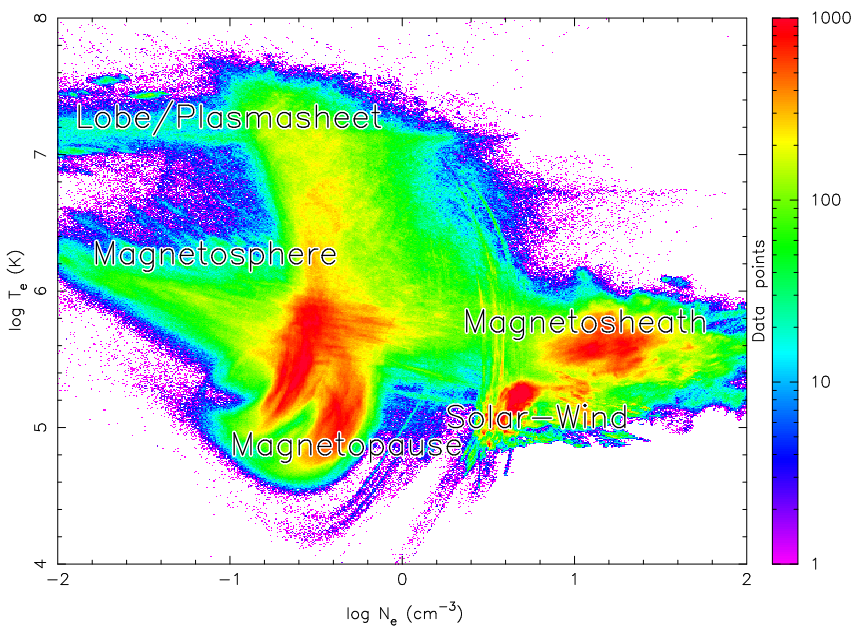

Fig. 3. Data map of a survey of all four Cluster spacecraft over the duration of 2002 PEACE operations: approximately $1.5 \times 10^{7}$ data points contribute to each map. White regions indicate lack of coverage - a more complete survey will result in a more detailed map. The total computer time required for these corrections was approximately 11 days. The colour in this map corresponds to the number of points per $\left(N_{f}, T_{f}\right)$-bin showing the overall coverage of the survey, labelled roughly according to magnetospheric region.

no correction is required (no such critical point exists in general for the temperature or velocity). In this circumstance the energy range truncation (resulting in an under-sampled distribution) is compensated by the potential broadening caused by $\Phi_{\text {crit }}$ such that, despite truncation and the presence of a potential, the density integration over $f(v)$ returns the correct value.

\section{Overview of results}

Cluster traverses a diverse range of environments, allowing PEACE to sample a wide range of parameter space. Consider the parameter space defined by $\left(N_{f}, T_{f}\right)$. We have corrected PEACE on-board calculated moments from all four of the Cluster spacecraft over the duration of 2002 scientific operations. This is an extensive survey, but small gaps in the data remain due to, for example, data availability, failure of the solver, etc. Plotting the distribution of various parameters in $\left(N_{f}, T_{f}\right)$-space provides a map of those parameters in terms of the plasma environment. In the context of this paper, Fig. 3 shows the overall coverage of the survey in this space together with the corresponding regions of the magnetosphere. In total, approximately $1.5 \times 10^{7}$ data points contribute to each map, requiring a total of 11 days of $\mathrm{CPU}$ (2.66 GHz, 4 GB RAM) time.

Theoretically, if the correcting algorithm is successful then no features of the potential (Fig. 4) should appear in the maps of corrected parameters, since the corrector aims to find free-space values which are unperturbed by spacecraft effects. This appears to be the case. For example, Fig. 5 shows the distribution of the bulk corrected velocity 


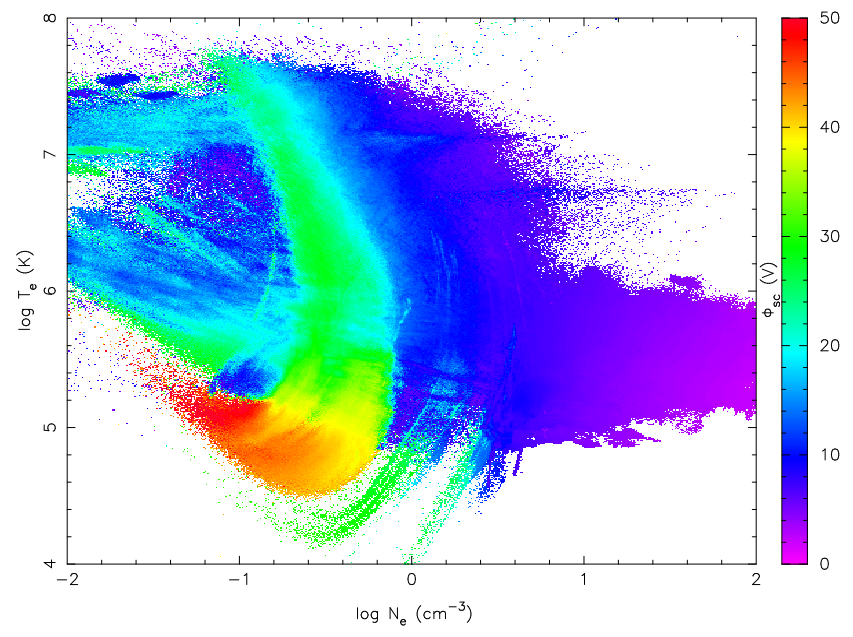

Fig. 4. Format as in Fig. 3 showing the spacecraft potential in $\left(N_{f}, T_{f}\right)$-space (if the EFW probe potential was unavailable, we used a constant spacecraft potential of $7 \mathrm{~V}$ ).

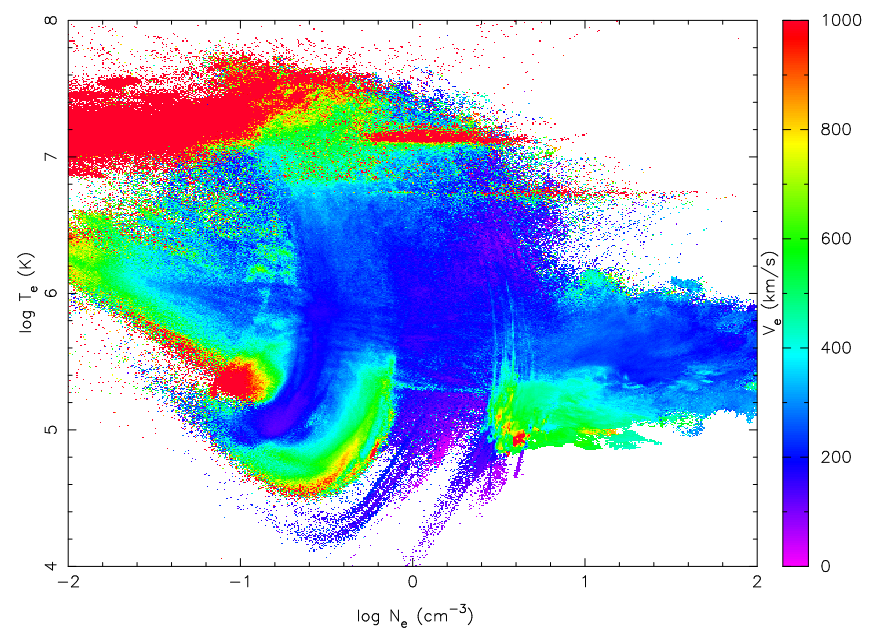

Fig. 5. Format as in Fig. 3 showing the bulk corrected velocity magnitude $V_{f}$.

magnitude $V_{f}$. The main features are the high-velocity streams (i.e. $V_{f}>1000 \mathrm{~km} / \mathrm{s}$ ) at $T_{f} \gtrsim 10^{7} \mathrm{~K}$, and swathes of relatively high-velocity electrons amongst a generally low majority $\left(V_{f}<200 \mathrm{~km} / \mathrm{s}\right)$. Note that the well-covered region at $N_{f}>1 \mathrm{~cm}^{-3}$ shows a high- $V_{f}$ structure at around $T_{f} \sim 10^{5} \mathrm{~K}$ (corresponding to the solar wind), which disappears at around $N_{f}>10 \mathrm{~cm}^{-3}$ and $T_{f}>5 \times 10^{5} \mathrm{~K}-$ the potential throughout this region is stable at about $10 \mathrm{~V}$. Similarly the swathe of high potential (the shape of which is likely due to the data coverage) at low densities does not seem to influence the corresponding region of the velocity map.

We now turn to more specific datamaps of the various estimation parameters to assess the degree to which the raw and corrected parameters differ. These maps should be considered against Fig. 3 which shows the data coverage, or more precisely, the number of data-points which contribute to each $\left(N_{f}, T_{f}\right)$-bin. Figures 6-9 show the variables $r_{N}, r_{V}, r_{T}, I_{e}$

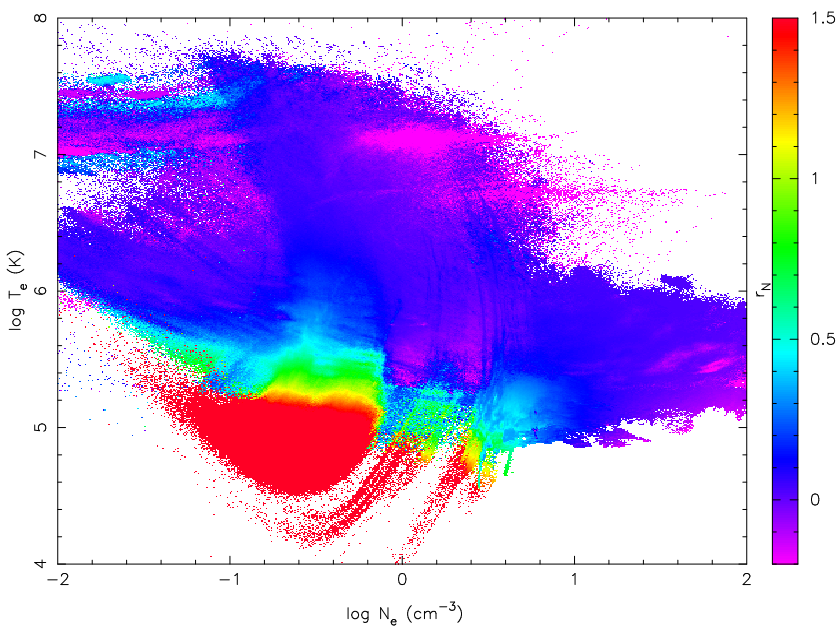

Fig. 6. Format as in Fig. 3 showing the density estimation parameter $r_{N}$. The value of $r_{N}$ indicates the fractional correction applied to the density moment. Positive(negative) values indicate that the onboard moments are over(under)-estimates of the true (i.e. corrected) moments.

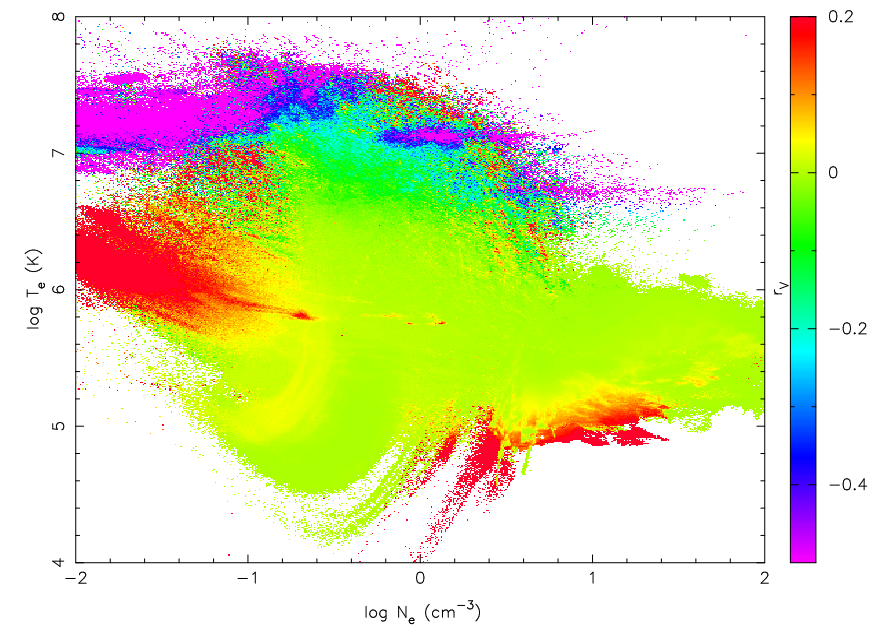

Fig. 7. Format as in Fig. 3 showing the velocity estimation parameter $r_{V}$ (see Fig. 6 caption).

in the space constrained by $\left(0.01 \leq N_{f} \leq 100 \mathrm{~cm}^{-3}\right)$ and $\left(10^{4} \leq T_{f} \leq 10^{8} \mathrm{~K}\right)$.

Figure 9 plots the global deviation $I_{e}$ of the on-board moments to the true moments. Comparing this with maps of other variables such as bulk velocity and spacecraft potential, we can gain an overview of the interplay between many of the dominant factors involved in the correction process over many of the regions Cluster encounters. In the case of $I_{e}$ (Fig. 9), for $\left(10^{6} \lesssim T_{f} \lesssim 10^{7} \mathrm{~K}\right)$, the overall correction is generally less than $20 \%$ for all densities. In contrast, for $T_{f} \lesssim 10^{6} \mathrm{~K}$ and $N_{f} \rightarrow 0 \mathrm{~cm}^{-3}$, the moments become severely affected and the overall correction required rapidly exceeds $300 \%$ - this is dominated by the over-estimation of the density and temperature moments (velocity in general is not so badly affected in this region) these individual patterns can be seen in the maps for $r_{N}, r_{T}, r_{V}$ (Figs. 6-8). 


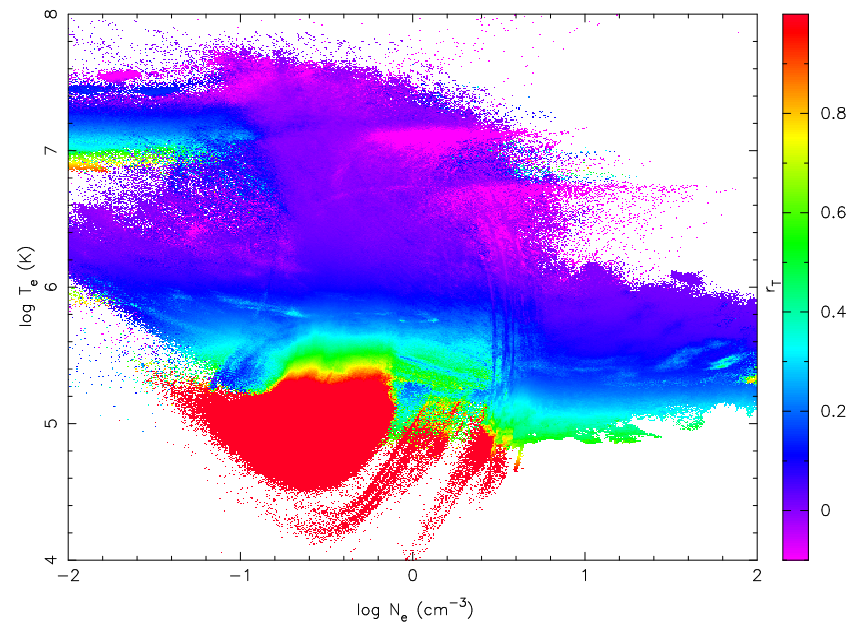

Fig. 8. Format as in Fig. 3 showing the temperature estimation parameter $r_{T}$ (see Fig. 6 caption).

To complement the survey, we simulate the behaviour of the general estimation parameter $I_{e}$ by passing a model truncated Maxwellian distribution through the algorithm with a bulk velocity range $\left(10 \leq V_{\text {sim }} \leq 1000 \mathrm{~km} / \mathrm{s}\right)$ which has been shifted with potentials over the range $\left(0 \leq \Phi_{s c} \leq 30 \mathrm{~V}\right)$. The truncation in energy was between $10 \mathrm{eV}$ and $1 \mathrm{keV}$, in accordance with previous simulations (e.g. GS) and typical PEACE operating parameters. The simulated estimation parameter is a minimal value since the effects of calibration defects are not dealt with, but which in reality contribute to the accuracy of the on-board moments. The results of this simulation are shown in Fig. 10 revealing an insensitivity to density, since the normalised inputs to the non-linear system (see the Appendix) are independent of $N_{m}$. Furthermore, as the temperature of the plasma increases, a significant portion of the distribution function is missed, since it lies beyond the upper integration limit, therefore we expect at around $T_{f} \sim 10^{7} \mathrm{~K}$ (corresponding to $E_{U} \sim 1 \mathrm{keV}$ ) the overall correction required will increase - this results in a banded structure to the simulation map.

In the $I_{e}$ distribution, we should expect to see strong correlation with the potential, and while there is correspondance at low- $\left(N_{f}, T_{f}\right)$ in Fig. $9, I_{e}$ is dominated by a band of low-correction which is almost independent of density (as shown in Fig. 10), and constrained around $\left(10^{6} \leq T_{f} \leq 10^{7} \mathrm{~K}\right)$. The low-correction band is situated such that sufficient range of the distribution is sampled and so only a slight correction is required, and this appears to be consistent even for $\Phi_{s c} \gtrsim 20$ V. By contrast, in the simulation reported in Fig. 10 $\Phi_{s c}$ and $V_{\text {sim }}$ are scanned uniformly over parameter space). The rapid increase in $I_{e}$ at high potentials is consistent with the findings of GS, who simulate $r_{N}$ for potentials exceeding the lower energy cut-off in various regions. To a lesser extent, for $T_{f} \gtrsim 10^{7} \mathrm{~K}, I_{e}$ rises to around $100 \%$ in this hightemperature/low-density region (e.g. the lobe/plasmasheet). The reason for the rise in $I_{e}$ in this high-temperature regime is due to the truncation in energy at the high-end of the range, which is typically between $1-2 \mathrm{keV}$ as expected from the $I_{e}$

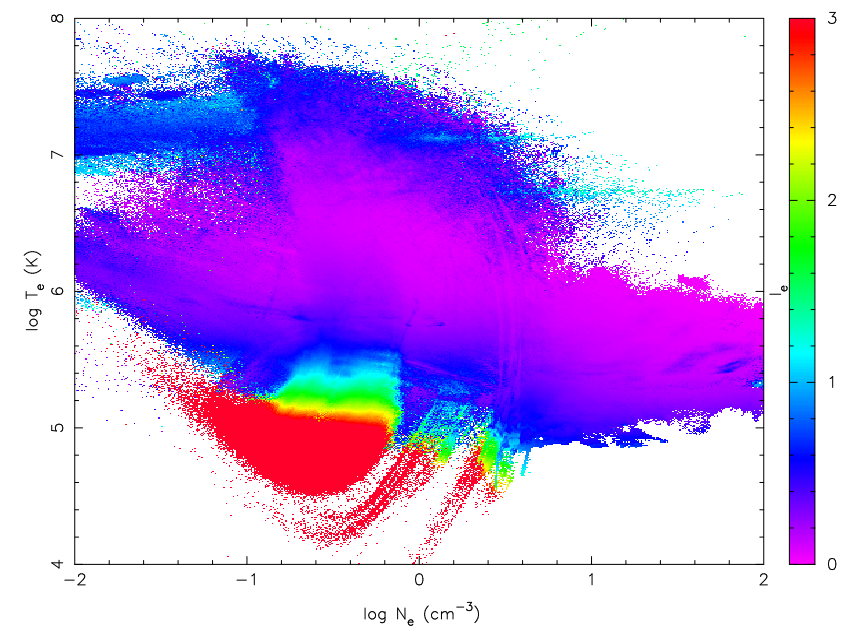

Fig. 9. Data map of global estimation parameter $I_{e}$. The value of $I_{e}$ indicates the magnitude of the fractional correction applied to all three on-board calculated moments.

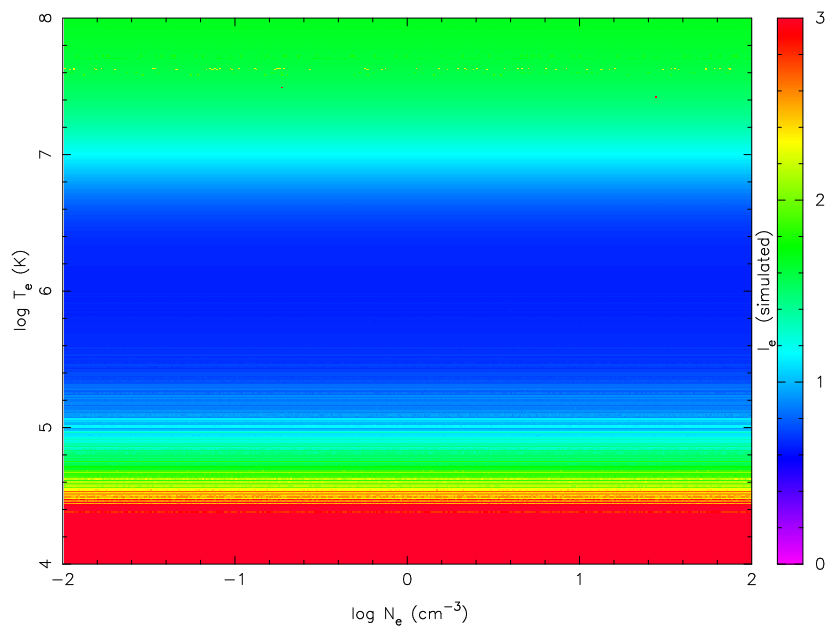

Fig. 10. Simulation of $I_{e}$ from a truncated, potentially broadened model Maxwellian distribution (see text for precise details of simulation).

simulation. The strong correction required at low densities and temperatures is dominated by the presence of a potential (which tends to be high compared to $E_{L}$ in this regime), whereas as the temperature increases and the extra broadening by $\Phi_{s c}$ becomes neglibible, it is the upper cut-off which becomes important, since large portions of the population are missed.

The data maps can only give an overall impression of the behaviour of the algorithm, since the exact outcome can depend on a number of factors which are averaged out of the maps. Such factors could include the precise mode of the detector, or unusual plasma characteristics which do not conform to the parameter space we present. Nevertheless, the mapping exercise provides a useful guide which can be built on to give a general model which helps us to better understand, and what to expect from, on-board moment calculations. 


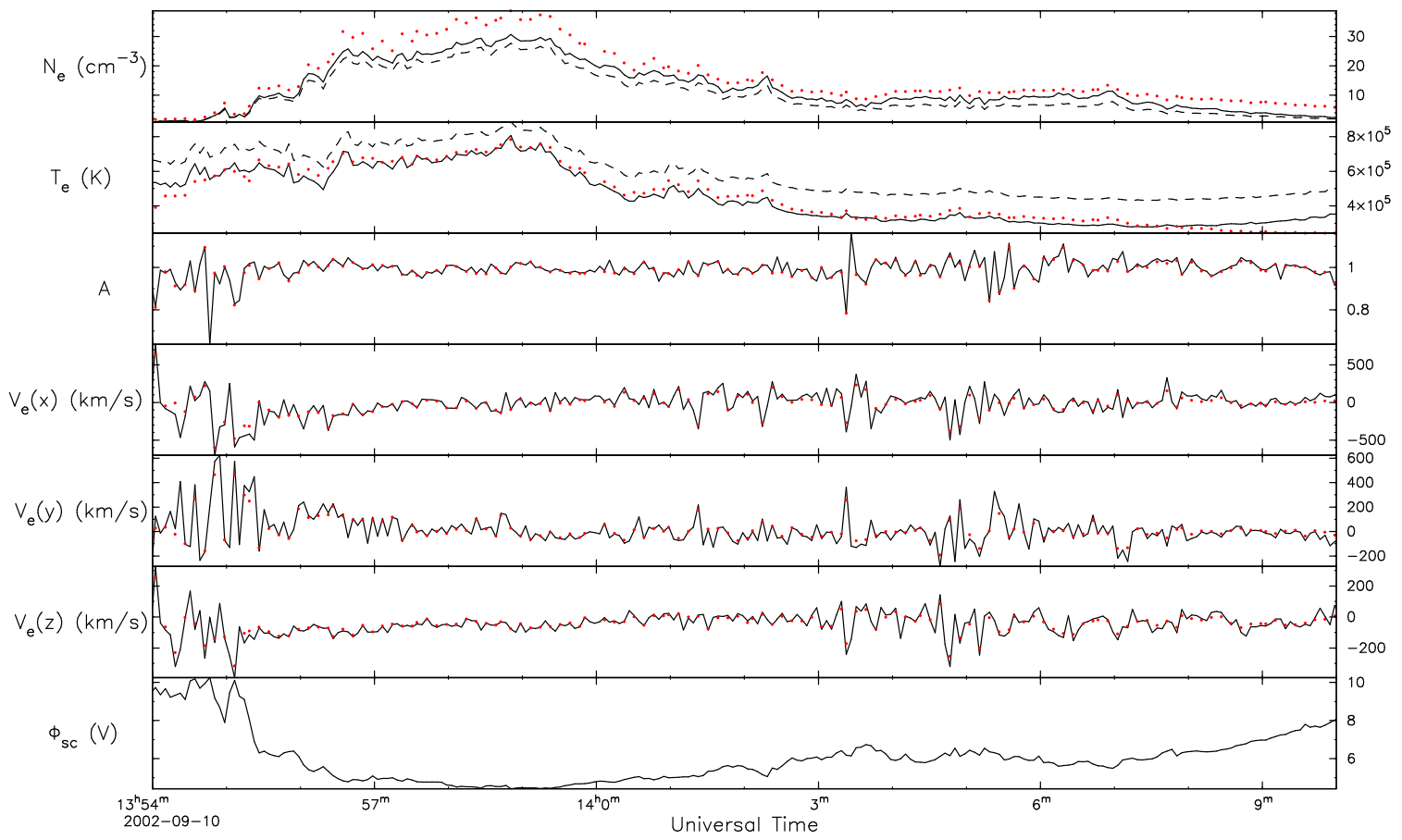

Fig. 11. Comparison between ground 3-D moments (dotted red trace), corrected moments (black trace), and un-corrected on-board moments (dashed black trace). These data were observed by Cluster 1 on 10 September 2002 in the cusp. The velocity in this case required little correction and we do not plot the un-corrected values. In addition to the moments we plot the temperature anisotropy $A$ and spacecraft potential.

\section{Comparison with ground calculations and other in- struments}

When telemetry allows, full 3-D distribution data can be transmitted. Integrations are then performed on the ground using a more sophisticated technique than is available onboard, such that the potential shift can be corrected before the moment sum takes place. These integrations scan over individual energy bins and angles (which are telemetered in discrete azimuthal zones), and in addition, a more detailed calibration model can be applied. Compared to the procedure presented in this paper, in which the distribution has to be inferred from its moments, 3-D ground moments represent the most accurate technique to extract moment data from spacecraft. Despite the benefits of the ground calculation, they are only intermittently available, since the 3-D data requires much more bandwidth than the transmission of the few numbers which encode all the basic information about the distribution - the on-board moments - which are available more frequently. Here we discuss the comparison between the results of the methods.

\subsection{Density}

Density is most sensitive to the presence of a potential, and typically requires the most correcting. In addition to the truncation and potential broadening of the distribution, care must be taken to avoid contamination from photo-electrons, which contribute to the number count on-board the spacecraft to varying degrees, depending on the environment. Our method does not deconvolve this contamination analytically, however we attempt to minimise the problem by comparing the lower energy cut-off $E_{L}$ with the spacecraft potential $\Phi_{s c}$ (as described in Sect. 2.2). Lower energy moments (i.e. the B moments) are not included if $\Phi_{s c}$ falls within a threshold limit $\Delta \Phi$ of the lower energy cut-off $E_{L}$, such that the B moments are not included if $\Phi_{s c} \geq E_{L}-\Delta \Phi$ (in all the examples shown, $\Delta \Phi=0 \mathrm{~V})$. This has the drawback of excluding a significant portion of phase-space, since eliminating the B moments typically removes natural electrons also. This leads to errors in the final moments under conditions for which the excluded population has different characteristics to those at overlap and higher energies. Note that this technique is not possible when the detectors are in complete overlap mode (see Fig. 2), in which case there are no B moments, so the lower energy cut-off is fixed at around $10 \mathrm{eV}$ irrespective of the value of $\Phi_{s c}$. The 3-D calculation applies a similar, but more precise, method by only removing electrons from a certain number of energy bins which are above the spacecraft potential. This has the benefit of only eliminating electrons which are likely to be photo-electron contaminants, and minimising the elimination of natural electrons.

The major difference between the ground 3-D treatment and the correction procedure is the handling of the unsampled region of $f(v)$ below the lower velocity cut-off. In the 3-D calculation, the moment sum can be tailored to a certain range of energy bins, such that $v_{l}^{3 \mathrm{D}}$ tends to be lower than 


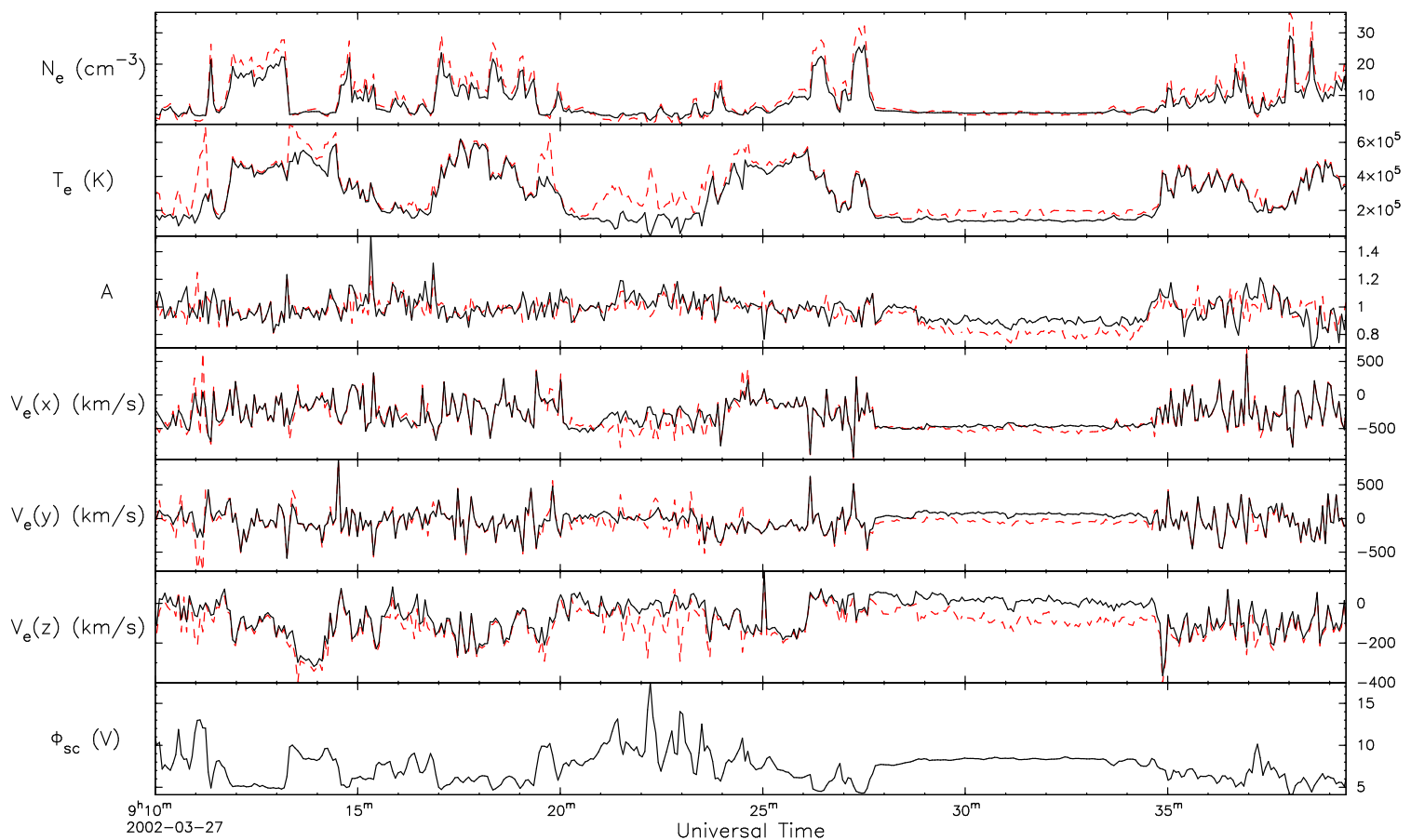

Fig. 12. Comparison between ground 3-D moments (dashed red trace) and corrected moments (black trace). These data were observed by Cluster 1 on 27 March 2002 in the magnetosheath and solar wind. There is generally good agreement in this interval, with only slight disparities in, for example the temperature, corresponding to spikes in the spacecraft potential (e.g. 0920-0925).

the $v_{l}$ used in the on-board calculation, since the potential is subtracted before the integration. For the corrected moments however, the algorithm fills the unsampled phase-space with a model Maxwellian distribution, fit from the truncated region, which we assume represents the nature of the ambient plasma. On this premise, we expect a slight disparity between not only the density results, but the other moments too, since the non-linear system involves functions of all three moments.

It is also possible that the correction method we employ does not fully correct the on-board moments at low potentials because of our under- or over-estimation of the spacecraft potential. $\Phi_{s c}$ is determined by empirically correcting the EFW (Electric Fields and Waves) probe value of the potential

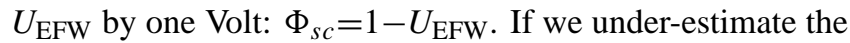
potential then we will typically under-correct the moments, and vice versa; however this potential difference estimate becomes less important at higher $\Phi_{s c}$ (note, the 3-D calculation is also susceptible to such an error).

In Fig. 11 we show a specfic example of a time-series of the un-corrected on-board calculated moments in comparison to the corrected ones and the 3-D derived results. The main correction required is in the density and temperature (little correction is required for the velocity, and for clarity we have not shown the on-board trace here). The density was under-estimated and the temperature over-estimated the correcting algorithm has improved the on-board values to concur with the 3-D ground calculations. The temperature is in near perfect agreement, and while the corrected density is good, it is still somewhat lower than the 3-D density. We discuss reasons for these differences in the next section.

Figures 12-14 (top panels) show density results from the two methods from different plasma environments: Figs. 12, and 14 show results predominantly from the magnetosheath, but also the less dense solar wind. Figure 13 is from the plasmasheet. In Fig. 14 we also plot results from two other Cluster instruments. WHISPER (Waves of HIgh frequency and Sounder for Probing of Electron density by Relaxation) experiment (Décréau et al., 1997) infers the electron density from the plasma frequency, providing a reliable and unambiguous measurement in the range $\left(0.25 \leq N_{\mathrm{WHI}} \leq 80 \mathrm{~cm}^{-3}\right)$; and the CIS (Cluster Ion Spectrometry) experiment (Rème et al., 2001), which measures the hot ion distribution, from which some measure of the electron density can be inferred. In Fig. 14, Cluster is in the magnetosheath, prior to exiting into the solar wind near the end of the plot. We choose the $N_{\mathrm{WHI}}$ as a reference, and plot the ratios $N_{f} / N_{\mathrm{WHI}}$, $N_{3 \mathrm{D}} / N_{\mathrm{WHI}}$ and $N_{\mathrm{CIS}} / N_{\mathrm{WHI}}$ in the bottom panel. Generally all three ratios are close to unity, indicating instrument (and method)-wide agreement, suggesting that any of these techniques could be used to gain an accurate value for the density. The mean difference in this interval $\left(N_{3 \mathrm{D}}-N_{\mathrm{WHI}}\right)$ is $+3.4 \mathrm{~cm}^{-3}$, and for $\left(N_{f}-N_{\mathrm{WHI}}\right)$ the mean difference is $-2.3 \mathrm{~cm}^{-3}$.

As well as the correctable effects, at low-density the limitations of the detector itself cause problems as the 


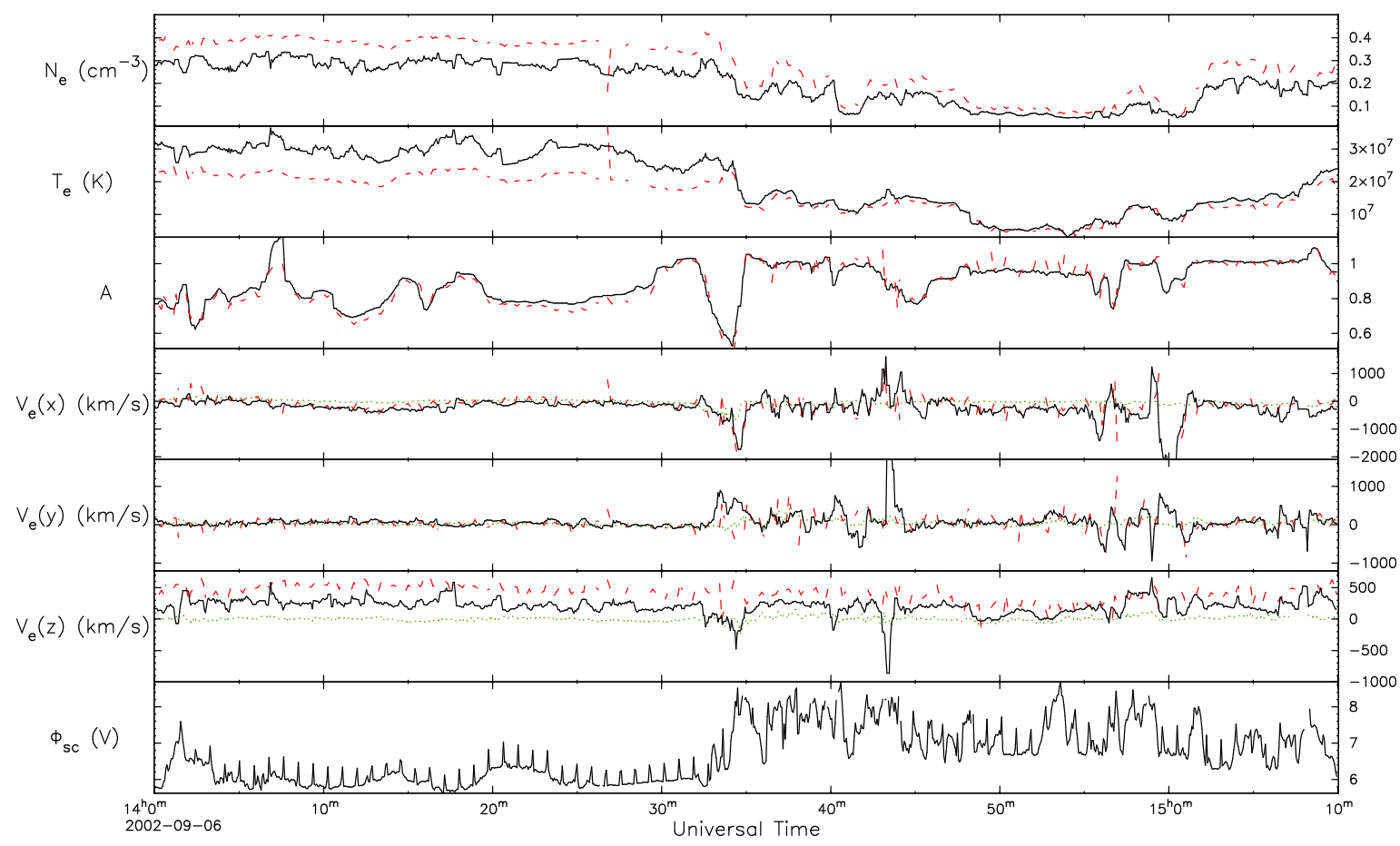

Fig. 13. Comparison between the electron moments (ground 3-D moments (dashed red trace) and corrected moments (black trace)), observed by Cluster 3 on 6 June 2002 in the plasmasheet. Note that periodic spikes in the spacecraft potential caused by WHISPER sounding propagate into the on-board and hence the corrected moments, therefore for clarity the corrected moment traces have been smoothed with a 30-s wide boxcar. The 3-D moments have not been smoothed. Also shown are components of the CIS (HIA) derived velocity (green dotted trace). The low-density conditions make an accurate density measurement difficult, but there is good agreement on the velocity vector, despite the high temperature. After the jump in potential at around 14:35 UT, there is excellent agreement between the temperature moments. In this interval the low-energy B moments had to be discarded since they contain a non-Maxwellian population dominated by photo-electrons which adversely affect our correction.

signal-to-noise ratio falls and the charge of the spacecraft increases, so the corrected moments in regions such as the plasmasheet (e.g. Fig. 12) must still be used with caution. It is the temperature, however, which is the major factor governing the outcome of the algorithm. For hot distributions, the extra broadening of the distribution (e.g. Fig. 1) by the spacecraft potential and truncation is negligible compared to the nominal width. Conversely, broadening of cooler distributions has a non-negligible effect, and we expect that in general more correction will be required as the temperature falls. Furthermore, as $N_{f} \rightarrow 0 \mathrm{~cm}^{-3}$, there are insufficient ambient electrons to balance the current flow of liberated electrons from the spacecraft surface, hence (without control) $\Phi_{s c}$ will build up to potentials exceeding $50 \mathrm{~V}$. It has been shown with active potential control experiments that the excessive charging of the spacecraft can be constrained to acceptable levels (i.e. $\left.\Phi_{s c}<10 \mathrm{~V}\right)$, and while improvements in detector technology may be made (e.g. in sensitivity and energy range coverage), the natural characteristics of the plasma one is trying to measure will always place a limit on the accuracy of the on-board moments.

\subsection{Temperature}

Generally, the overall correction of the temperature is small $\left(\left|r_{T}\right|<0.2\right)$, but increases as $T_{f}$ becomes comparable to the upper and lower energy cut-off (see Fig. 8 and discussion of $I_{e}$ in Sect. 5.1). Cluster encounters a range of (electron) temperatures which typically range from $\left(10^{4} \lesssim T_{f} \lesssim 10^{8} \mathrm{~K}\right)$, which is within the operating capabilities of the correcting algorithm. In the magnetosheath and solar wind (Figs. 12 and 14), the corrected temperature generally agrees well with the 3-D derived temperature. In some parts of the hot plasmasheet the two are nearly identical (Fig. 13). As mentioned above, for disparities between the density results of the two methods, we expect to see differences of a similar magnitude in the temperature since the correcting algorithm uses a function of all three of the moments. Indeed, the behaviour of the correction required for the temperature is very similar to the density. For example, in Figures $6\left(r_{N}\right.$ map) and $8\left(r_{T}\right.$ map) both parameters indicate large overestimation in the low-density and low-temperature region, lesser correction required in the $\left(10^{6}<T_{f}<10^{7} \mathrm{~K}\right)$ band, and regions of under-estimation at higher temperatures. The fact that the temperature can be under-estimated by the on-board 


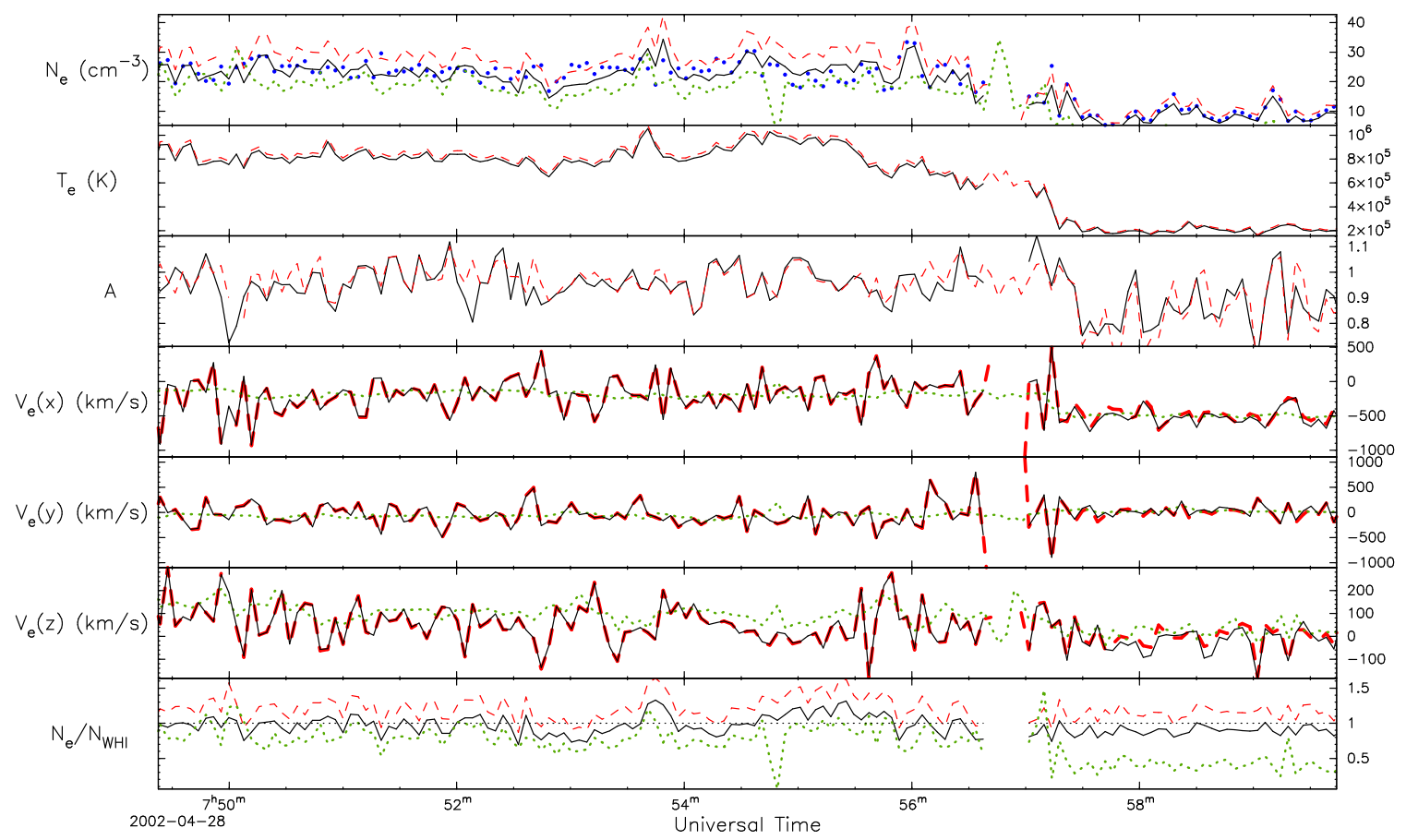

Fig. 14. Comparison between ground 3-D moments (dashed red trace), corrected moments (black trace), WHISPER density (dotted blue trace) and CIS data (dotted green trace). These data were observed by Cluster 1 on 28 April 2002 in the magnetosheath until the spacecraft crossed the bow-shock just after 0757. The bottom panel shows the ratio of the various densities and highlights the success of our correcting algorithm. Note the WHISPER data has been averaged to spin-resolution.

calculation is interesting since it suggests that in certain regions the upper-energy cut-off is too low to sample the significant high-energy population.

\subsection{Velocity}

Generally, the magnitude of the velocity requires little correction as evidenced in Fig. 7. This is not true in the solar wind and lobe/plasmasheet regions, where the overall correction $I_{e}$ can be dominated by $r_{V}$. At high-temperature/lowdensity (lobe/plasmasheet) the velocity tends to be underestimated since the sum starts to miss portions of the distribution beyond the upper cut-off, and at low-temperature/highdensity the broadening caused by the spacecraft potential results in an over-estimation. The major difference in the behaviour of $r_{V}$ (Fig. 7) compared to the other moments is the lack of correction required at low-density and lowtemperatures. In this region the density and temperature require the most correcting, but the velocity requires little. Instead, for $T_{f}<10^{5} \mathrm{~K}$ the velocity is over-estimated as the density increases into the solar wind region, and for $T_{f}>10^{7} \mathrm{~K}$ is under-estimated for the lobe/plasmasheet region.

Our assumption that the direction of the velocity vector is not changed by the spacecraft potential appears to be a good approximation, since the corrected and 3-D results are in good agreement - note all velocity data shown is in the GSE frame. In the case of the plasmasheet region (Fig. 13) we found that to get good agreement, we had to systematically discard the low-energy B moments, since they appear to contain a population of photo- or secondary electrons which result in a large disparity in the velocity result caused by erroneous inclusion of these electrons which are returning to the detector. Spacecraft-related electrons are particularly problematic in regions where the ambient density is low.

\subsection{Temperature anisotropy}

To estimate the temperature anisotropy $A$, we assume that $T_{\perp} / T_{\|}$is not affected by the spacecraft potential. We then contract the measured pressure tensor with the direction of the magnetic field (using data from Cluster's Fluxgate Magnetometer (FGM) experiment (Balogh et al., 2001) as described in Sect. 3.4. Compared to the anisotropy derived from the 3-D ground calculation, this appears to be an effective strategy since both techniques are in excellent agreement (e.g. Figs. 12 and 13).

\section{Limitations}

The technique presented here provides an efficient method to correct on-board calculated moments from the dominant spacecraft effects of non-zero potential and energy range truncation to an estimate of their true values. The simplicity of the algorithm afforded by virtue of several underlying assumptions (e.g. isotropic Maxwellian distribution, scalar 
approximation, etc.) also introduces several limitations, of which some could be improved upon in future:

- The present model does not fully accommodate the effects of contamination from photo- and secondary electrons which become a dominant problem when the spacecraft potential exceeds the lower energy cut-off. Grard (1973), for example, proposes a model of a photoelectron distribution as a bi-Maxwellian distribution with component thermal energies of $\sim 2 \mathrm{eV}$ and $\sim 7 \mathrm{eV}$.

- Given the numerical nature of the algorithm, another model distribution function could be used instead of the drifting Maxwellian we employ.

- We do not model the non-spherical aspects of the spacecraft potential, which could improve accuracy, but at the cost of abandoning the scalar approximation which simplifies analytical handling. Indeed, in general particle trajectories are not purely radial even in a sphericallysymmetric potential,

- The myriad sources of calibration defects all contribute to the overall over- and under-estimation of the on-board moments. The instrument-specific details (energy-binning, gain corrections, etc.) require userintervention to incorporate their overall effect into the on-board moment corrector.

- It would be possible, at the cost of considerable added complexity, to pass a model distribution through a full detector response simulation to replace the functions of $g_{i}$ in Eqs. (11-13) in the inversion algorithm.

\section{Summary of numerical method}

The algorithm presented here can be summarised as follows:

1. The measured moments $\left(N_{m}, V_{m}, T_{m}\right)$ can be described as functions of the moments $(N, V, T)$ of an idealised drifting Maxwellian, the velocity cut-offs $v_{l, u}$ and the spacecraft potential $\Phi_{s c}$. The measured moment function triplet must be inverted to derive the values of the Maxwellian moments.

2. A normalised non-linear system is set-up and primed with a set of three initial guesses which are to be iteratively improved using a Newton-Raphson algorithm.

3. For speed and numerical accuracy, we treat the measured high energy $\left(v>v_{u}\right)$ partial moments in a separate stage, whereby we do not correct them, but sum them with the lower energy corrected moments.

4. The direction of the velocity vector can be determined from the input velocity moment vector, as we make the scalar assumption to simplify the calculation, whereby only the magnitude of the particle velocities, and hence also the thermal velocity are affected by potential.
5. To estimate the anisotropy, we contract the measured pressure tensor $\mathbf{p}_{m}$ with the unit magnetic field vector $\mathbf{b}$ to extract the ratio $A_{m}=p_{m \perp} / p_{m \|}$. We can then partition the final temperature $T_{f}$ into parallel and perpendicular components: $3 T_{f}=T_{f \|}+2 T_{f \perp}$ so that $A_{m}=T_{f \perp} / T_{f \|}$.

\section{Conclusions}

In this paper we have demonstrated the implementation of the procedure proposed by Génot and Schwartz (2004). Onboard moments must be corrected because they convolve spacecraft effects such as a non-zero potential and finite energy cut-offs, as well as the effect of numerous calibration defects. We have tested the correcting procedure extensively on data taken by Cluster's PEACE experiment (though the algorithm is generic), and shown that the corrected moments compare well with ground based calculations and data from other Cluster instruments, demonstrating that on-board calculated moments can be corrected to gain accurate data from orbital detectors, despite the physical limitations of flying such devices in space. This has significant benefits, since the 3-D ground moment calculations are not available as frequently as the on-board moments, which provide substantial high-resolution data coverage. Thus, by implementing the procedure we present here, the operational capabilities of existing and future experiments such as PEACE can be fully exploited to yield useful scientific results which might otherwise be lost.

\section{Appendix The normalised non-linear system}

We normalise the unknown moments in terms of the measured ones as:

$$
\begin{aligned}
& V_{c}^{\prime}=\frac{\left|\boldsymbol{V}_{c}\right|}{V_{m}} \\
& V_{T c}^{\prime}=\frac{V_{T c}}{V_{T m}} \\
& \eta=\frac{1}{\sqrt{\pi}} \frac{N_{c} / N_{m}}{V_{c}^{\prime} V_{T c}^{\prime}} .
\end{aligned}
$$

where $V_{T c}$ is the corrected thermal speed, from which the temperature can be inferred:

$T_{c}=\frac{m_{e} V_{T c}^{2}}{2 k}$

The inputs to the solver are the normalised quantities

$$
\begin{gathered}
\zeta_{s c}=\frac{V_{m}}{V_{T m}} \\
\epsilon_{s c}=\frac{\mathcal{E}}{V_{T m}^{2}} \\
V_{L, U}=\frac{v_{L, U}}{V_{T m}}
\end{gathered}
$$


For the inputs $v_{L}=0, v_{U}=\infty, \mathcal{E}=0$, the exact solution yields unity for Eqs. (A1, A2) and $1 / \sqrt{\pi}$ for Eq. (A3).

The set of non-linear equations which must be inverted are:

$g_{1}\left(N_{c}, V_{c}, T_{c}\right)-N_{m}=0$

$g_{2}\left(N_{c}, V_{c}, T_{c}\right)-N_{m} V_{m}=0$

$g_{3}\left(N_{c}, V_{c}, T_{c}\right)-3 N_{m} k T_{m}-m_{e} N_{m} V_{m}^{2}=0$

That is, the measured moments are functions of the real moments. We recast to a normalised system such that we wish to find the triplet $\left(\eta, V_{c}^{\prime}, V_{T c}^{\prime}\right)$, given the normalised inputs $\left(\zeta_{s c}, \epsilon_{s c}, V_{L, U}\right)$. The equations to be solved are

$$
1-\frac{\eta}{\zeta_{s c}} \int_{V_{L}}^{V_{U}} \sqrt{V^{2}-\epsilon_{s c}}\left(E^{-}-E^{+}\right) \mathrm{d} V=0
$$

$$
\begin{array}{r}
\zeta_{s c}^{2}-\eta \int_{V_{L}}^{V_{U}}\left(V^{2}-\epsilon_{s c}\right)\left[E^{-}+E^{+}\right. \\
\left.-\frac{V_{T c}^{\prime 2}\left(E^{-}-E^{+}\right)}{2 V V_{c}^{\prime} \zeta_{s c}}\right] \mathrm{d} V=0
\end{array}
$$

$$
\begin{aligned}
\frac{3}{2}+\zeta_{s c}^{2}-\frac{\eta}{\zeta_{s c}} \int_{V_{L}}^{V_{U}} & \left(V^{2}-\epsilon_{s c}\right)^{3 / 2} \\
& \times\left(E^{-}-E^{+}\right) \mathrm{d} V=0
\end{aligned}
$$

where

$$
E^{ \pm}=\exp \left[-\left(\frac{V \pm V_{c}^{\prime} \zeta_{s c}}{V_{T c}^{\prime}}\right)^{2}\right]
$$

Acknowledgements. We wish to thank the CIS team for the use of ion data in the verification of our algorithm. We also acknowledge the support of the FGM team for access to magnetic field data; the EFW team for the use of the probe potential and the Hungarian Data Centre who supply attitude information, allowing transformation of data into the GSE frame. All these sources were vital in the success of this work. JG was supported by a grant from the UK Particle Physics and Astronomy Research Council.

Topical Editor T. Pulkkinen thanks R. Treumann and another referee for their help in evaluating this paper.

\section{References}

Balogh, A., Carr, C. M., Acuña, M. H., Dunlop, M. W., Beek, T. J., Brown, P., Fornacon, K.-H., Georgescu, E., Glassmeier, K.H., Harris, J., Musmann, G., Oddy, T., and Schwingenschuh, K.: The Cluster Magnetic Field Investigation: Overview of in-flight performance and initial results, Ann. Geophys., 19, 1207-1217, 2001,

\section{SRef-ID: 1432-0576/ag/2001-19-1207.}

Décréau, P. M. E., Fergeau, P., Krasnosels'kikh, V., Lévêque, M., Martin, Ph., Randriamboarison, O., Sené, F. X., Trotignon, J. G., Canu, P., and Mogensen, P. B.: Whisper, a Resonance Sounder and Wave Analyser: Performances and Perspectives for the Cluster Mission, Space Sci. Rev., 79, 157-193, 1997.

Escoubet, C. P., Pedersen, A., Schmidt, R., and Lindqvist, P. A.: Density in the magnetosphere inferred from ISEE 1 spacecraft potential, J. Geophys. Res., 102, 17 595-17 610, 1997.

Génot, V., and Schwartz, S. J.: Spacecraft potential effects on electron moments derived from a perfect plasma detector, Ann. Geophys. 22, 2073-2080, 2004.

Grard, R. J. L.: Properties of the satellite photoelectron sheath derived from photoemission laboratory measurements, J. Geophys. Res., 78, 2885-2906, 1973.

Johnstone, A. D., Alsop, C., Burge, S., Carter, P. J., Coates, A. J., Coker, A. J., Fazakerley, A. N., Grande, M., Gowen, R. A., Gurgiolo, C., Hancock, B. K., Narheim, B., Preece, A., Sheather, P. H., Winningham, J. D., and Woodliffe, R. D.: PEACE: a Plasma Electron and Current Experiment, Space Sci. Rev., 79, 351-398, 1997.

Pedersen, A.: Solar wind and magnetosphere plasma diagnostics by spacecraft electrostatic potential measurements, Ann. Geophys., 13, 118-129, 1995,

SRef-ID: 1432-0576/ag/1995-13-118.

Press, W. H., Teukolsky, S. A., Vetterling, W. T., and Flannery, B. P.: Numerical Recipes in Fortran 77: The Art of Scientific Computing, Cambridge University Press, 1992.

Riedler, W., Torkar, K., Rudenauer, F., Fehringer, M., Pedersen, A., Schmidt, R., Grard, R. J. L., Arends, H., Narheim, B. T., Troim, J., Torbert, R., Olsen, R. C., Whipple, E., Goldstein, R., Valavanoglou, N., and Zhao, H.: Active Spacecraft Potential Control, Space Sci. Rev., 79, 271-302, 1997.

Salem, C., Bosqued, J.-M., Larson, D. E., Mangeney, A., Maksimovic, M., Perche, C., Lin, R. P., and Bougeret, J.-L.: Determination of accurate solar wind electron parameters using particle detectors and radio wave receivers, J. Geophys. Res., 106, 9, 21 701-21 717, 2001.

Schmidt, R., Arends, H., Pedersen, A., Rudenauer, F., Fehringer, M., Narheim, B. T., Svenes, R., Kvernsveen, K., Tsuruda, K., Mukai, T., Hayakawa, H., and Nakamura, M.: Results from active spacecraft potential control on the Geotail spacecraft, J. Geophys. Res., 100, 17 253-17 260, 1995.

Song, P., Zhang, X. X., and Paschmann, G.: Uncertainties in plasma measurements: Effect of lower cutoff energy and spacecraft charge, Planet. Space Sci., 45, 2, 255-267, 1997.

Szita, S., Fazakerley, A. N., Carter, P. J., James, A. M., Trávnícek, P., Watson, G., André, M., Eriksson, A., and Torkar, K.: Cluster PEACE observations of electrons of spacecraft origin, Ann. Geophys., 19, 1721-1730, 2001,

SRef-ID: 1432-0576/ag/2001-19-1721.

Torkar, K., Riedler, W., Escoubet, C. P., Fehringer, M., Schmidt, R., Grard, R. J. L., Arends, H., Rüdenauer, F., Steiger, W., Narheim, B. T., Svenes, K., Torbert, R., André, M., Fazakerley, A., Goldstein, R., Olsen, R. C., Pedersen, A., Whipple, E., and Zhao, H.: Active Spacecraft Potential Control for Cluster - Implementation and First Results, Ann. Geophys. 19, 1289-1302, 2001. 